\title{
3D finite element analysis on crack-tip plastic zone
}

\author{
Shashidhar K. Kudari ${ }^{1 *}$ and Krishnaraja G. Kodancha ${ }^{2}$ \\ $I^{I^{*}}$ Research Centre, Department of Mechanical Engineering, SDM College of Engineering \& Technology, Dharwad-580002, Karnataka, INDIA \\ ${ }^{2}$ Research Centre, Department of Mechanical Engineering, BVB College of Engineering \& Technology, Hubli-580031, Karnataka, INDIA \\ "Corresponding Author: e-mail: s.kudari@rediffmail.com, Tel +91-0836-2447465, Fax. +91-0836-2464638
}

\begin{abstract}
This paper presents an effect of specimen thickness on crack-tip plastic zone shape and size. The shape of the plastic zone is estimated in 3D with the help of elastic and elastic-plastic finite element analyses. The results indicate that the shape of plastic zone does not correspond to classical "dog-bone shape" and the maximum plastic zone size occurs beneath the free surface, contrary to conventional shape. It is observed that for specimens with $B / W=0.5$, the ASTM requirement of specimen thickness to estimate $K_{I C}$, the plastic zone shape and size is much larger than the one obtained in 2D plane strain analysis. The results also show a significant change in the shape and size of plastic zone in elastic and elastic plastic conditions.
\end{abstract}

Keywords: finite element analysis, thickness effect, 3D plastic zone.

\section{Introduction}

The plastic deformation at the tip of a crack inhibits the build-up of high stresses, and in turn governs the initiation and growth of cracks in structural materials. The extent of plastic deformation, which delineates the elastic-plastic boundary in a loaded structural component, is commonly referred to as plastic zone. The standard ASTM procedures like ASTM E399-83, ASTM E813-87, etc. for determining fracture toughness criteria of materials require knowledge of the extent of plastic deformation occurring at the crack-tips for determining the specimen dimensions. The $K_{I C}$ (plane strain fracture toughness) test criterion requires a specimen thickness sufficiently large so that the crack front at the centre of the specimen should be under a state of plane strain. Hence, the studies related to crack-tip plastic zones are of fundamental importance for assessing fracture criterion of a material. The current emphasis in fracture mechanics is the constraint issue. The general discussion on the constraint effects, however, often lacks a clear definition of what is meant by constraint. Yuan and Brocks (1998) have considered that constraint literally is a structural obstacle against plastic deformation, which is induced mainly by geometrical and physical boundary conditions. With detailed finite element analysis they have also argued that constraint effects in a specimen depend on the plastic zone size. The systematic understanding of structural obstacle against the plastic deformation requires a detailed study of crack-tip plastic deformation in 3D to distinguish between in-plane constraint and the out-of-plane constraint (Yuan and Brocks, 1998). For a given in-plane configuration, the plane strain state describes highest possible out-of-plane constraint and generates the highest possible stress triaxility, where as the plane stress yields the lower limit. In most of the cases the in-plane and out-of-plane constraints are mixed in such a way that their effects cannot be separated (Yuan and Brocks, 1998). In order to study the details of these in-plane and out-of-plane constraint effects it is required to conduct detailed 3D FE analysis of fracture specimen.

According to the conventional concept, the crack-tip plastic zone across the thickness of the specimen is given by the so called "dog-bone" model, which assumes a state of plane strain inside the specimen (centre) and a state of plane stress on the surface of the specimen as shown in Fig.1. In order to understand the theoretical variation of plastic zone size from surface to center of the specimen, 3D finite element analyses are required. Recently, it is found that 3D plastic zone FE models have been studied by Zhang et al. (1992), Roychoudhury and Dodds (2003), Fernandez-Zuniga et al. (2005), Gonzalez-Herrera et al. (2006) and Subramanya et al. (2007). But the detailed analysis of shape and size, comparison with 2D plane stress and strain models are lacking. An attempt has been thus directed in this investigation to achieve a few numerical estimations of 3D plastic zone shape and size. Primarily, these estimations have been made on single edge notched tensile (SENT) specimens having different plate 
thickness. In addition, attempts have been also made to estimate plastic zone shape and size using both elastic and elastic-plastic FE analyses.

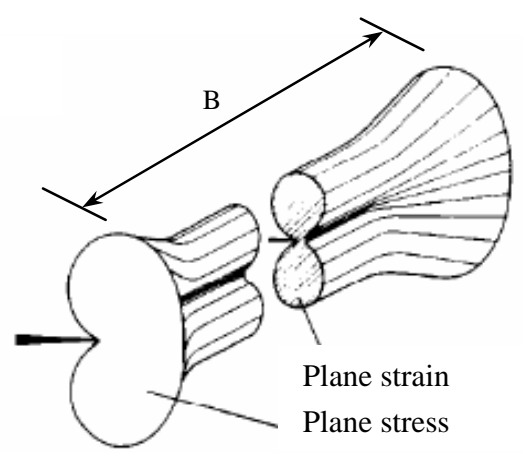

Figure 1. Schematic representation of 3 Dimensional plastic zone.

\section{Finite element analysis}

A series of 3D stress analyses by finite element method have been made on SENT specimen using ABAQUS 6.5-1 (2004) finite element software. The geometry of the specimen considered in these analyses is shown in Fig.2. Finite element computations were carried out considering only one half of the specimen due to their symmetry. Initially, two dimensional (2D) plane stress and plane strain analyses have been carried out to study the effect of plane stress and plane strain state of stress (condition) on plastic zone. In 2D analyses the analysis domain of interest is descritized using 1430 eight noded elements. 3D FE analyses have been conducted to study the effect of specimen thickness on shape and size of crack-front plastic zone and, $K_{I}$ and $J$-integral. The analysis domain is descritized using 20-noded isoparametric 3D solid reduced integration elements. This kind of elements is used in the work of Qu and Wang (2006). The number of elements used in the analysis varied with the thickness of the specimen. A typical FE mesh used in the analysis for thickness $B=8 \mathrm{~mm}$ is shown in Fig.3.

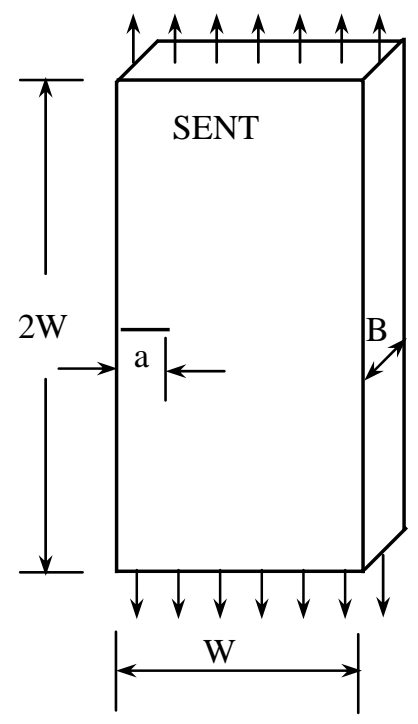

Figure.2. The geometry of the specimen.

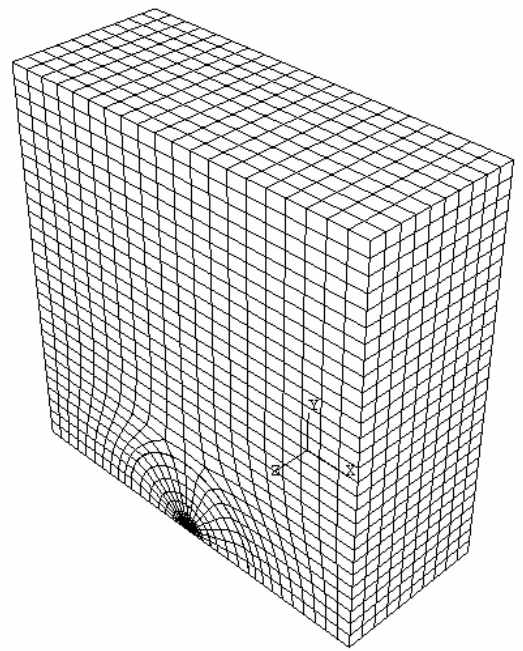

Figure.3. A typical FE mesh used in the analysis for thickness $B=8 \mathrm{~mm}$

The 3D shape and size of plastic zone and the magnitudes of $K_{I}$ and $J$ were obtained by ABAQUS post processor. The variation of plastic zone, $K_{I}$ and $J$ along the crack front have been studied for varied specimen thickness $(B)$ and crack length to width ratio, $a / W=0.5$. The normal applied stress $(\sigma)$ on the specimen is considered $=50 \mathrm{MPa}$, which is $1 / 3 \mathrm{rd}$ of yield stress $\left(\sigma_{y}\right)$ to keep the analysis domain approximately under LEFM. The specimen thicknesses $(B)$ considered in this analysis is varied from 2 to $20 \mathrm{~mm}$ $(B / W=0.1-1.0)$. The boundary separating the plastic enclave from the elastic bulk was obtained by iso-stress surface of the effective stress using von Mises yield criterion (Goudouts and Papakalitakis, 1987). In these FE analyses, the material considered is an interstitial free (IF) steel possessing yield strength $\left(\sigma_{y}\right)$ of $155 \mathrm{MPa}$, Poisson's ratio $(v)=0.3$ and elastic modulus $(E)$ of $197 \mathrm{GPa}$ as used in the work of Kudari et al. (2007). In case of elastic-plastic finite element analyses material deformation is considered as multilinear kinematic hardening type. The Newton-Rapson procedure in which stiffness matrix is updated for every equilibrium iteration was used for the nonlinear convergence. The material response in plastic deformation has been modeled by taking twenty 
divisions of plastic portion of true stress - true strain curve of the material as used in the work of Kudari et al. (2007). The plastic deformation between two successive points in the model was assumed to be linear with a particular tangent modulus. This multilinear model is used in this FEA is illustrated in Fig.4.

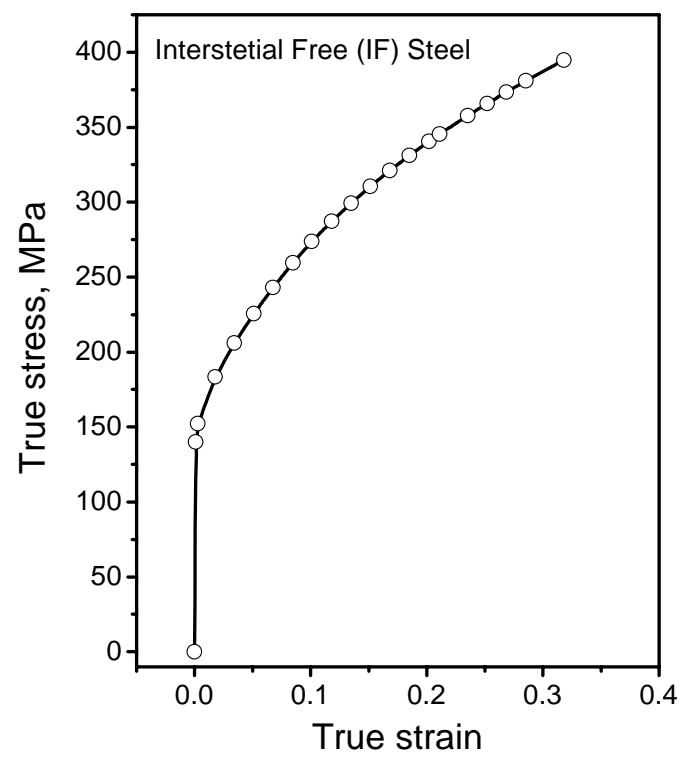

Figure.4. The multilinear material model used in FEA

\section{Results and Discussion}

Initially, 2D FE elastic and elastic-plastic plane stress and plane strain analyses have been carried out at applied stress level of $50 \mathrm{MPa}$ to study the effect of such ideal conditions on the plastic zone shape. The shape of the plastic zone ahead of a crack-tip has been ascertained by plotting the iso-surfaces of the effective stress, which causes yielding as per von Mises criterion. The plastic boundaries in a SENT specimen obtained by 2D FE elastic and elastic-plastic analyses under plane stress and plane strain conditions are overlapped as illustrated in Fig.5(a) and Fig.5(b) respectively. Figure 5(b) indicates that the size of the plane stress plastic zone ahead of crack-tip is significantly larger than the one obtained by elastic analysis (Fig.5(a)). On the other hand the size of the plane strain plastic zone estimated by elastic-plastic FEA is considerably smaller than the one obtained by elastic FEA. The varied shape in 2D plastic zone shapes is attributed to the effect of plasticity used in the FEA. These 2D results are used to compare the 3D plastic zones at the centre (mid plane of the specimen) and on the surface of the specimen.
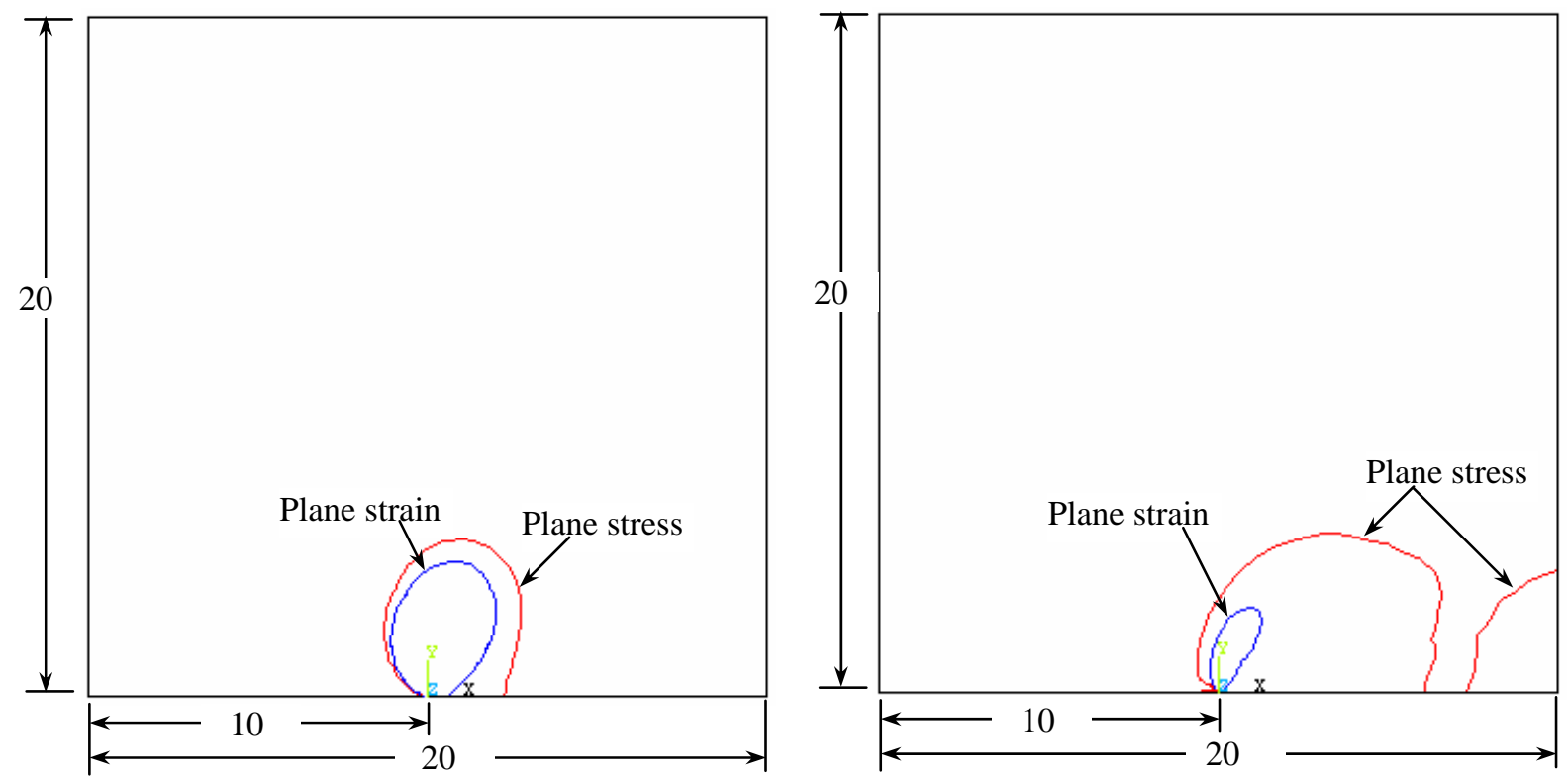

Figure 5. Plastic zone shape in a SENT specimen obtained by 2D FEA (a) elastic analysis (b) elastic-plastic analysis (Dimensions of specimen are in $\mathrm{mm}$ ) 
A series of 3D FE stress analyses have been carried out on SENT specimen with varied thickness $(B)$ and $a / W=0.5$ to study the plastic zone , variation of stress intensity factor, $K_{I}$, and $J$-integral along the crack-front for constant applied stress level of $50 \mathrm{MPa}$ $\left(\sigma / \sigma_{y}=0.30\right)$. The 3D elastic-plastic boundaries in specimens having thickness $B=2,6,10,15$, and $20 \mathrm{~mm}$ (pertaining to $B / W=0.1$ to 1) obtained by elastic FE analysis are shown in Fig.6. Similarly, shapes of plastic zones obtained using elastic-plastic FE analysis for various thickness of specimen are shown in Fig.7. The results in Fig.6 show that the shape and size of plastic zone in 2 $\mathrm{mm}$ thick specimen corresponds, practically, to that of 2D plane stress results (Fig.5 (a)) throughout the thickness. It is observed that the sizes as well as the shape of surface PZ change for specimens of higher thicknesses. This result indicates that the actual state of stress on the free surface of the specimen gets changed as specimen thickness is increased, and is no more represented by a state of a plane-stress. The crack-front plastic zones at the mid-plane of the specimen, changes with increasing thicknesses of the specimen and, slowly converges to the size and shape similar to the state of plane strain. The 3D shapes of PZ (Fig.6) along the crack-front across the thickness of the specimen show a composite behavior in the region below the specimen surface. For B $<6 \mathrm{~mm}$ it is observed that the PZS is bigger at the mid-plane than on the surface of the specimen. On the other hand, for $B>6 \mathrm{~mm}$, when seen from the mid plane to the specimen surface, the plastic zone shows a gradual increase in the size, as already observed by Buchholz et al. (2004). But just before approaching the surface, PZ shows a sudden decrease in size, very different from the behavior predicted by the dog-bone model. Hence, the results clearly demonstrate that the shape of plastic zone obtained by elastic FEA does not correspond to the dog-bone (Fig.1).
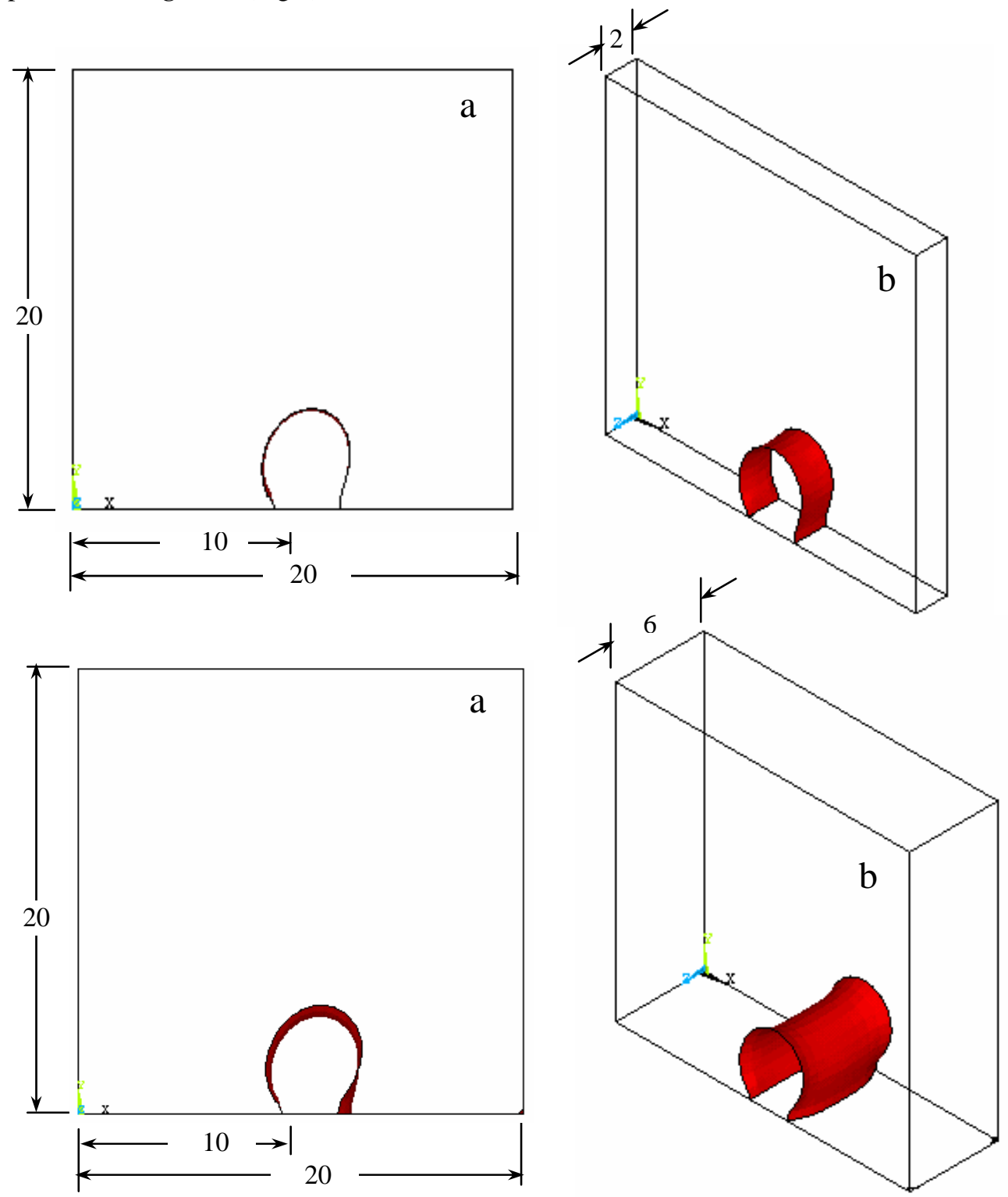

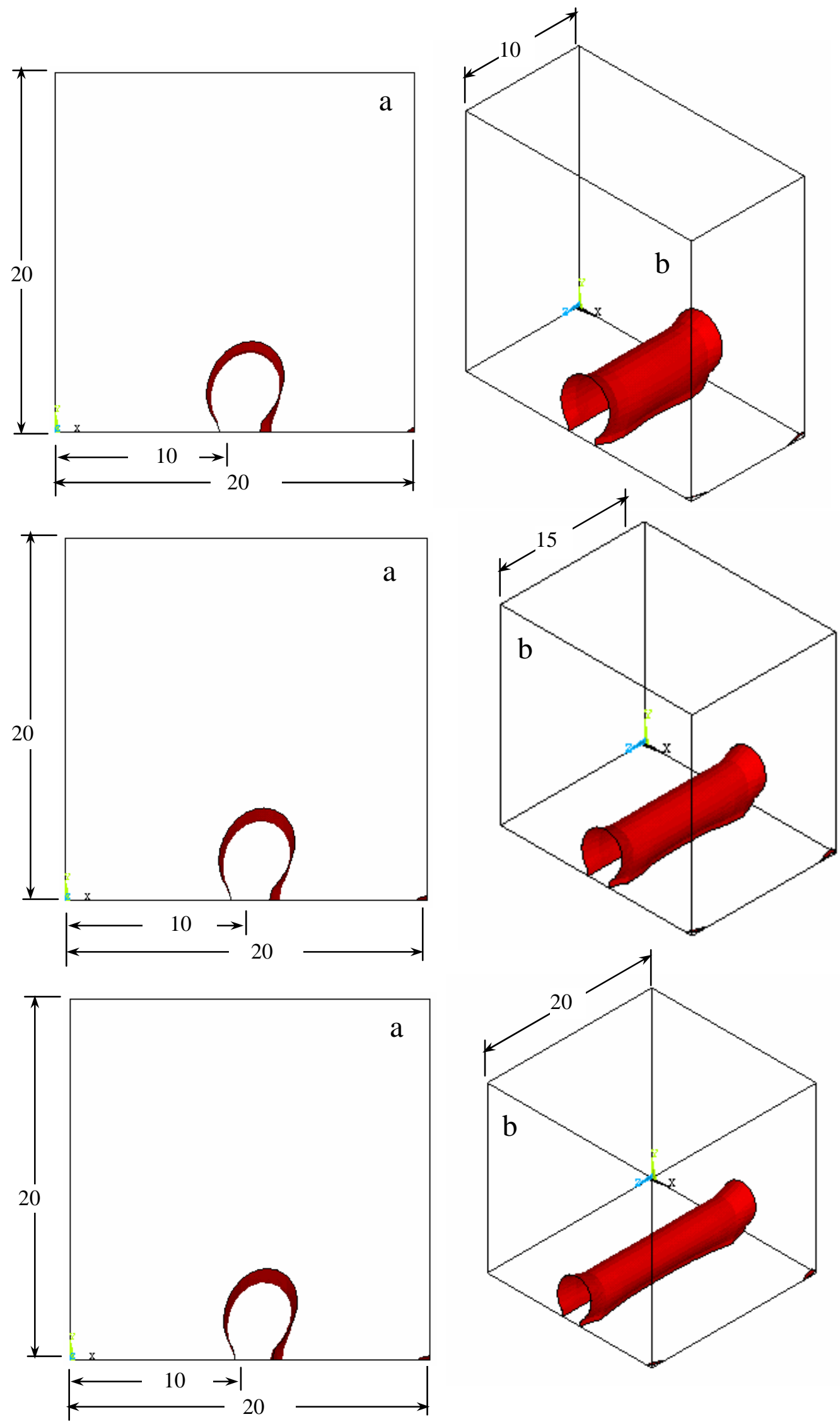

Figure 6. Plastic zone shape in a SENT specimen having varied thickness obtained by elastic FEA (a) front view and (b) isometric view (all dimensions are in $\mathrm{mm}$ ) 

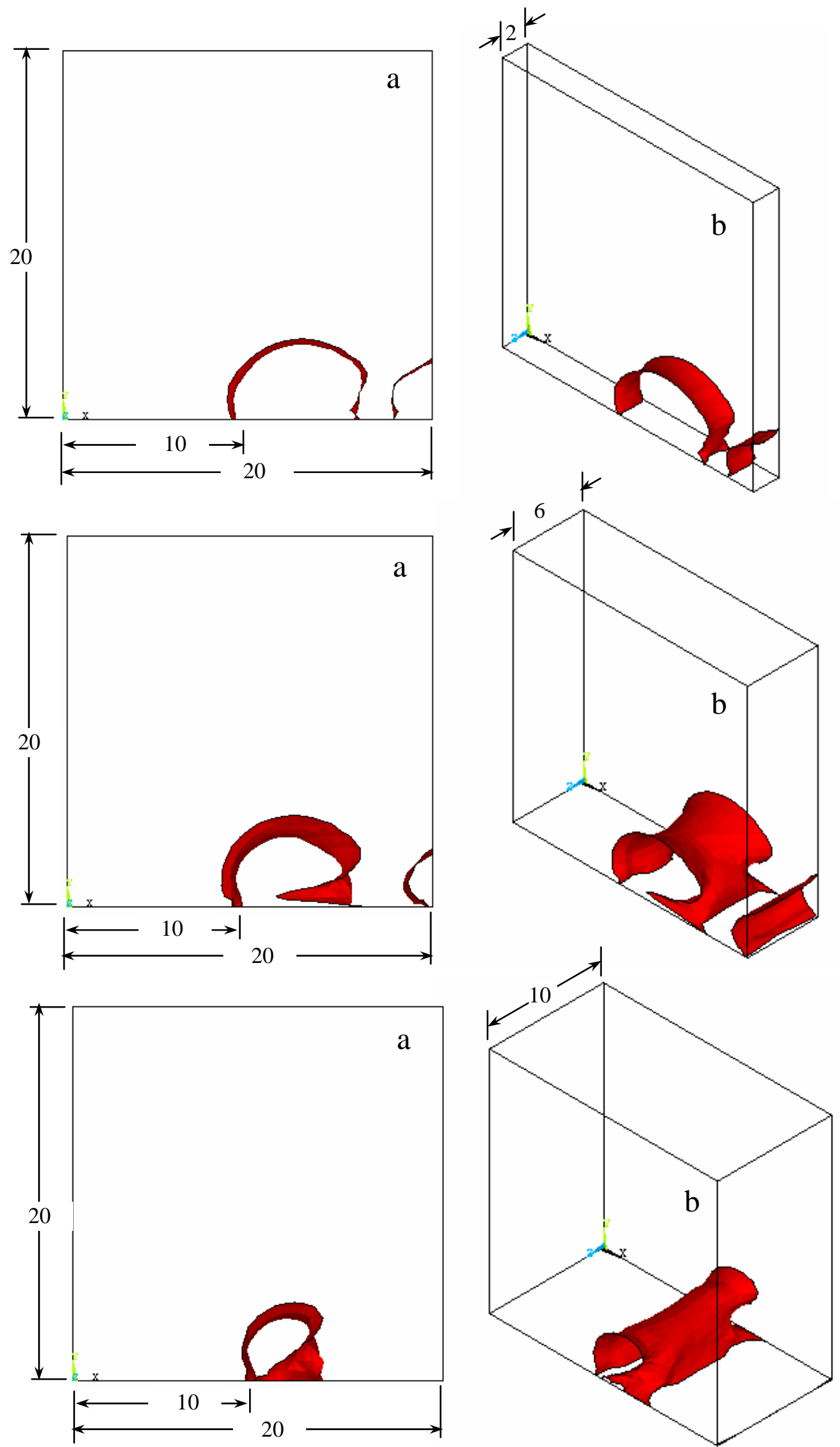

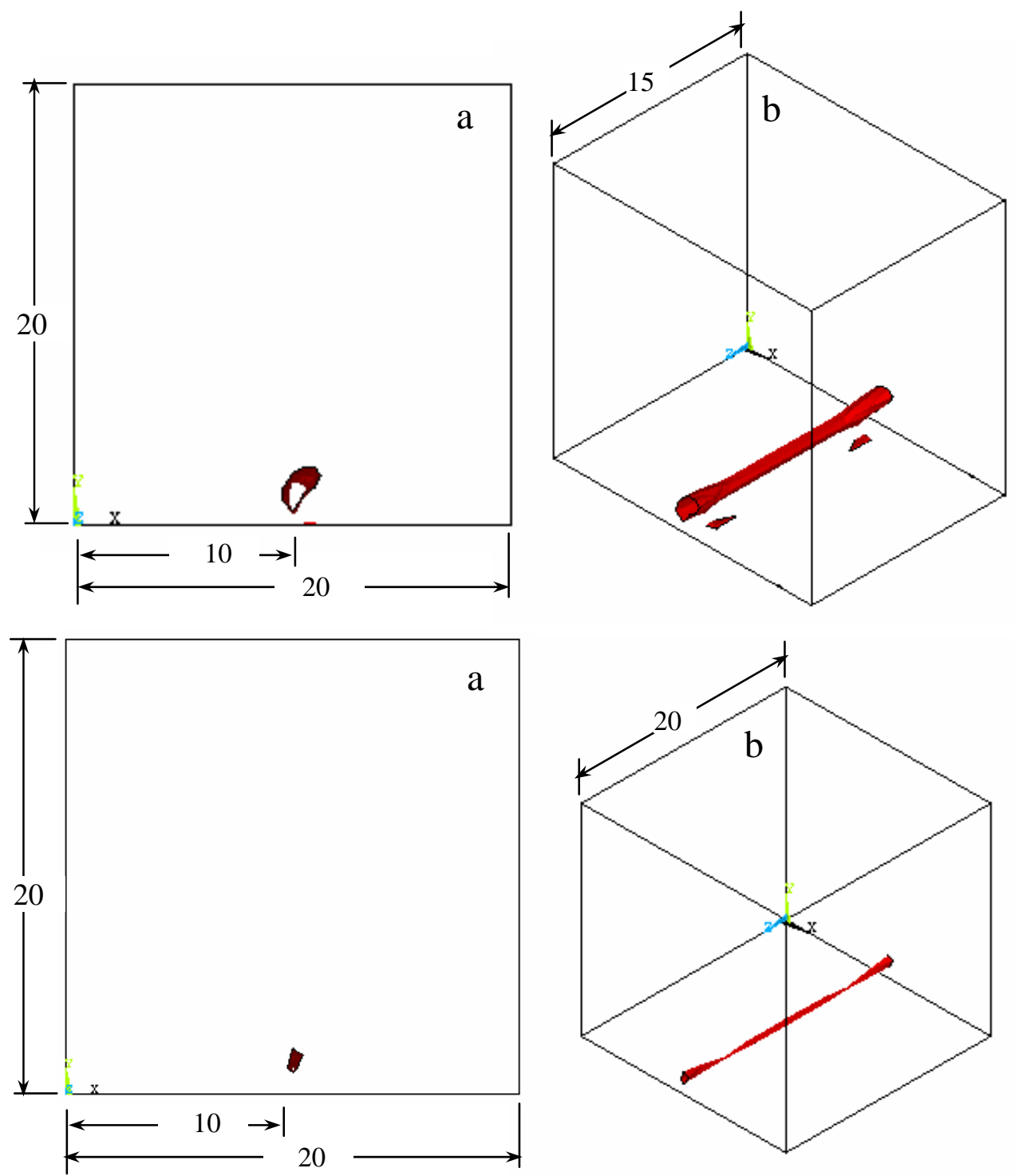

Figure 7. Plastic zone shape in a SENT specimen having varied thickness obtained by elastic-plastic FEA (a) front view and (b) isometric view (all dimensions are in $\mathrm{mm}$ )

The smaller size of the plastic zone in specimen interior according to the elastic FEA results, see Fig.6, indicate that the in-plane and out-of-plane constraints are mixed in such a way that their effects are complex to study because of occurrence of larger plastic zone size beneath the surface.

The results corresponding to the elastic-plastic analysis depicted in Fig.7 show that the shape of the plastic zones in specimens of different thickness is dissimilar in nature. The shape and size of plastic zone in $2 \mathrm{~mm}$ thick specimen is almost similar to that of 2D plane stress results. It is observed from the results that the shape of the plastic zone on the surface and at center of the specimen gradually changes from plane stress to plane strain state as the thickness of the specimen is increased. This elastic-plastic FE analysis indicates that for higher thick specimens there is no difference in in-plane and out-of-plane constraints. It is clearly studied that, in-plane effects vanish as thickness of the specimen increases, and only out-of-plane constraint effects dominate, indicating dominance of plane strain condition. Interestingly, it is observed that for $B=10 \mathrm{~mm}(B / W=0.5)$ specimen thickness as required by ASTM E399-83, the PZS has not reached the plane strain condition as compared to 2D plane strain result shown in Fig.5(a). It is observed that for approximately $B>12 \mathrm{~mm}(B / W=0.6)$ the shape of the plastic zone on the surface and at the center is very much similar to the 2D plane strain result. This observation is unlike the theoretical visualization of 3D plastic zone as shown in Fig.1. The Figures 6 and 7 clearly demonstrate that the shape and size of 3D plastic zone strongly depend on the plasticity model, and in both the cases (elastic and elastic-plastic) they differ from the classical dog-bone model.

The extent of plastic zone size, $r_{p}$, at $\theta=0^{\circ}$ on the surface and at the centre of the specimen has been estimated for a large number of plastic enclaves obtained by the FE analyses using two different approaches as shown in Fig.6 and Fig.7. The outputs of the 
plastic enclave obtained by the FE analyses were used to estimate the value of $r_{p}$ by two different approaches. The magnitude of $r_{p}$ was estimated using ABAQUS post processor by making displacement scaling equal zero and noting the nodal co-ordinates of the interest that were in contact or near to the effective stress contour possessing a value equal to the uniaxial yield strength of a material so long $r_{p}<2 \mathrm{~mm}$. In the second approach the magnitude of $r_{p}$ was measured by pixel count of the image using suitable calibrations for $r_{p}>2 \mathrm{~mm}$ (Kudari et al., 2007). The use of the first approach was convenient so long as the FE mesh size finer like that existed near the tip of a crack. The results of the extent of plastic zones were compiled as its size $r_{p} v s$. specimen thickness $B$ obtained using elastic and elastic-plastic FE analysis are shown in Fig.8 and Fig.9 respectively. The magnitudes of $r_{p}$ estimated by 2D plane stress and plane strain conditions are also shown in Fig.8 and Fig.9 by horizontal lines for comparison with 3D results. The ratio of plastic zone sizes on the surface and at the center of the specimen in elastic $\left(R_{e l}\right)$ and elastic-plastic $\left(R_{e l-p l}\right)$ FE analyses were examined and are shown in Fig.10 and Fig.11 respectively.

The estimated values for the plastic zone, $r_{p}$, for different specimen thickness are shown in Fig.8 indicating that the difference in the $r_{p}$ on the surface and at the center of the specimen varies with thickness. Figure 8 indicates that magnitudes of $r_{p}$ on the surface and at the centre of the specimen having thickness $2 \mathrm{~mm}$ almost match with 2D plane stress result as expected. But, the magnitude of $r_{p}$ at the centre of the specimen with higher specimen thickness $(B \geq 10 \mathrm{~mm})$ does not match with 2D plane strain result. According to ASTM E399-83 the specimen has to attain plane strain condition for specimen thickness, $B=0.5 W$ (ie. $B=10 \mathrm{~mm}$ ) for a valid $K_{I C}$ test, which is not observed even at $B=W=20 \mathrm{~mm}$ in this analysis. Figure 8 also shows that, for specimen thicknesses, $B<$ $8 \mathrm{~mm}$, the plastic zone size at the center of the specimen is higher than that on the surface, and vice versa for $B>8 \mathrm{~mm}$, this implies that using elastic analysis for estimation of the PZS in thin specimens $(B<8 \mathrm{~mm})$ is inappropriate. The ratio for plastic zone size on the surface and at the center of the specimen $\left(R_{e l}\right)$ has been examined for specimen thicknesses $>8 \mathrm{~mm}$ demonstrating that the ratio increases with the specimen thickness, as shown in Fig.10. The results of 3D elastic-plastic analysis of PZS as depicted in Fig.9 indicate that: (a) for $2 \mathrm{~mm}$ thick specimen, the $r_{p}$ is almost similar at the surface and at the center, in agreement with the 2D plane stress result. (b) the size of a plastic zone is higher on the surface than that at the center of a specimen for any $B$ in agreement with natural expectation, (c) the difference in the magnitude of $r_{p}$ on the surface and that at the center of the specimen increases with $B$ and (d) the magnitude of $r_{p}$ at the centre of the specimen obtained by 3D FEA approximately matches with the 2D plane strain results for $B=10 \mathrm{~mm}$. One can see from Figure 9 that the value of $r_{p}$ obtained by elastic-plastic FEA at the centre of the specimen reduces from plane stress to plane strain value as $B$ increases from 2 to $10 \mathrm{~mm}$. This nature of PZ is unlike that in elastic case. According to ASTM E399-83 the specimen has to attain plane strain plastic zone size for $B=10 \mathrm{~mm}(B / W=0.5)$ for the valid $K_{I C}$ fracture test. This condition is not observed from the results of elastic FEA even for $B=20 \mathrm{~mm}(B / W=1)$. But, interestingly, this condition is closely met for elastic-plastic condition. The ratio of the estimated plastic zone sizes at the surface and at the center (for elastic-plastic analysis), $R_{e l-p l}$, is examined in Fig.11. This figure indicates that $R_{e l-p l}$ increases as the thickness of the specimen increases. This ratio is found to be much higher than that of $R_{e l}$.

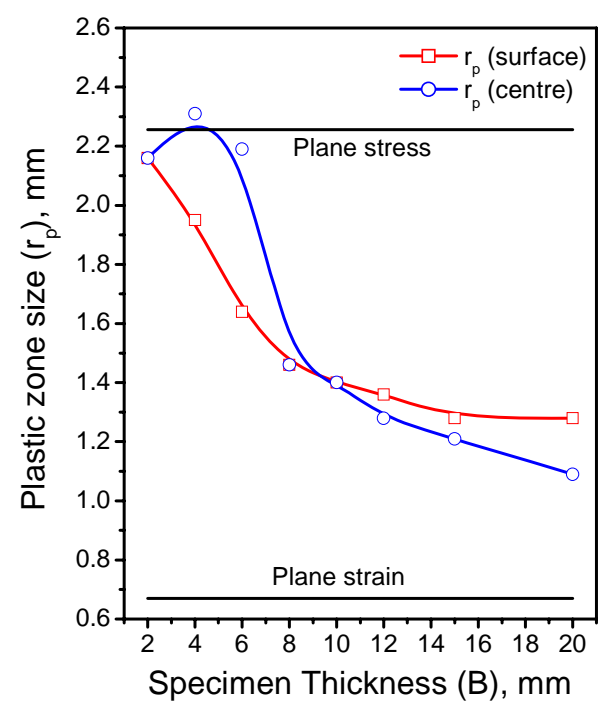

Figure 8. Variation of $r_{p}$ vs. $B$ by elastic FEA

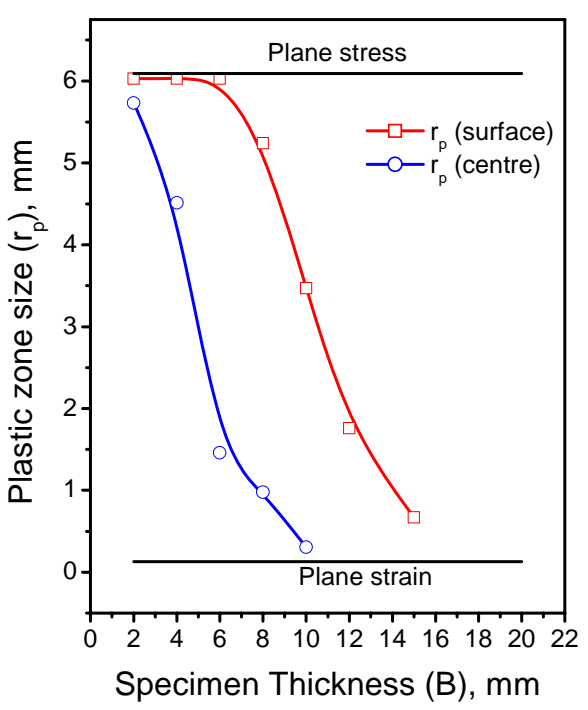

Figure 9. Variation of $r_{p}$ vs. $B$ by elastic-plastic FEA

Considering the state of stress at the center of the thick specimen to be plane strain and that on the surface to be plane stress, the shape and size of PZ have been obtained using both 2D FE elastic and 2D FE elastic-plastic analysis. These results are shown in Fig.5(a) and and Fig.5(b) respectively. The ratio of the estimated plastic zone sizes $(R)$ obtained by plane stress (on surface) and plane strain (at the center) 2-D elastic analysis (Fig.5(a)) is found to be 3.3 for $\theta=0^{0}$. This result is in good agreement with that predicted value ( 3.0) by Irwin's (1960) elastic analysis. The magnitudes of $R_{e l}$ estimated by 3D-elastic analysis, as shown in the 
Fig.10, increases with increasing specimen thickness, but do not yield any value closer to 3 within the range of $B=2-20 \mathrm{~mm}$ used in this computations. This indicates that 2D elastic analysis does not yield any proper results for the fracture analysis. The ratio of the estimated plastic zone sizes obtained by plane stress and plane strain 2D FE elastic-plastic analysis (Fig.5(b)), on the other hand, is found to be approximately 30. The result of similar nature is not available in the literature for any comparative assessment. The magnitudes of $R_{e l-p l}$ estimated by 3D FE elastic-plastic analysis, as shown in the Fig.11, is also found to increase with increasing specimen thickness, but the obtained results do not yield any value closer to 30 within the series of computations carried out for specimen thickness in the range 2-20 mm. It is seen from Figures 10 and 11 that the magnitudes $R_{e l}$ and $R_{e l-p l}$ are much smaller than that obtained by ratio of 2D plane stress and plane strain $r_{p}$ indicating the state of stress in a specimen is not exactly plane stress on the surface and plane strain at the centre of the specimen as usually considered in analytical analysis.

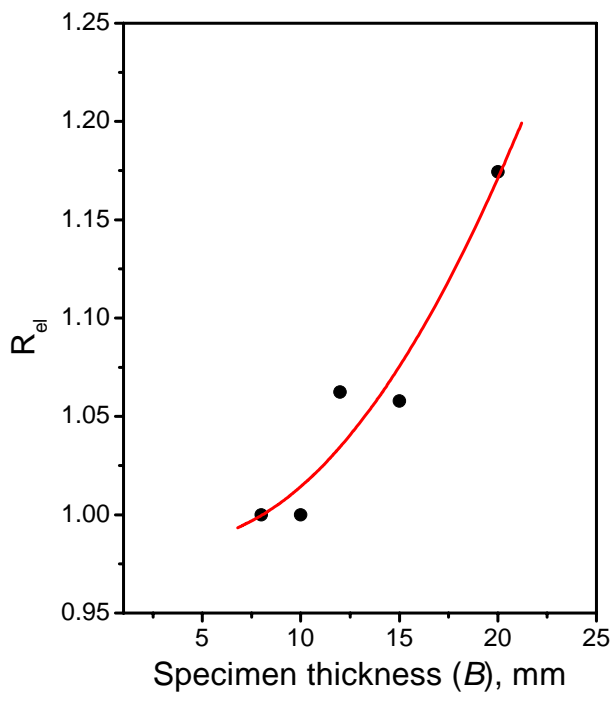

Figure 10. Variation of $R_{e l}$ vs. $B$ by elastic FEA

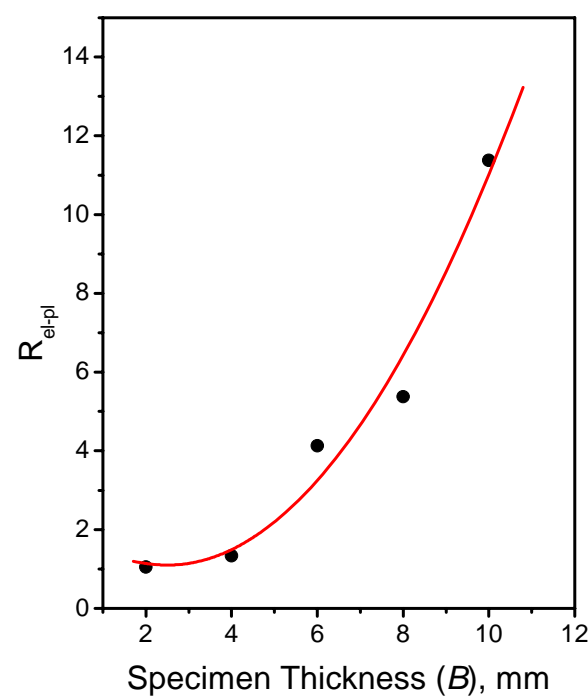

Figure 11. Variation of $R_{e l-p l}$ vs. $B$ by elastic-plastic FEA

In this work, the variation of stress intensity factor $\left(K_{I}\right)$ and $J$-integral along the crack-front obtained by elastic and elasticplastic analyses respectively are also studied. A typical plot of variation of $K_{I}$ along the crack-front for various specimen thicknesses and $a / W=0.5$ obtained by FE elastic analysis is shown in Fig.12. The magnitude of $K_{I}$ is estimated by ABAQUS post processor, the detailed discussions of extracting of $K_{I}$ can be found in Kodancha and Kudari (2009). Figure 12 indicates that the magnitude of $K_{I}$ is higher at the centre of the specimen than on the surface. The nature of variation of $K_{I}$ shown in Fig.12 is in good agreement with the similar results presented by Fernandez et al. (2005). The magnitude of $K_{I}$ estimated by 2D plane stress and plane strain conditions is found to be identical and is also plotted in Fig.12 by a horizontal line. Figure 12 shows that, the magnitudes of $K_{I}$ estimated by 3D FEA are specimen thickness dependent and are not comparable with 2D FEA results, as stated by Giner et al. (2010).

The magnitude of $J$-integral in 3D by FE elastic-plastic analysis has been computed in the similar manner it is computed in an earlier report (Coutin, 2005). The FE mesh (Fig.2) is divided into number surfaces equal to the number of elements along the thickness direction. By defining the nodes of the crack front, using domain integral method the software (ABAQUS) automatically finds the five contours (user defined) in order to carry out $J$-integrals. As it is widely accepted that the first contour does not provide good results because of numerical singularities (Coutin, 2005), the magnitude of first contours has been neglected in the analysis. The mean value of rest of four contours on each surface along the thickness direction is computed and typical such magnitudes of $J$ for various specimen thicknesses and $a / W=0.5$ are plotted in Fig.13. Figure 13 indicates that the magnitude of $J$ varies from surface to the centre of the specimen along the crack front. The magnitude of the $J$ is observed to be higher at the centre of the specimen than that of surface. The nature of variation of the magnitude of $J$ presented in Fig.13 is in similar agreement with the results presented in the work of Zadeh et al. (1995), Rajaram et al. (2000) and Giner et al. (2010). The magnitudes of $J$ estimated by 2D plane stress and plane strain cases are found to be different (unlike for stress intensity factor as shown in Fig.12) and are superimposed on Fig.13 for comparison with the 3D values of $J$-integral. It is observed that magnitude of plane stress 2D $J$-integral in close agreement with $J$ at the centre of $2 \mathrm{~mm}$ thick specimen and magnitude of plane strain 2D $J$ integral in close agreement with $J$ at the centre of $20 \mathrm{~mm}$ thick specimen.

These above discussed results indicate that thickness effect of the specimen is to be considered for estimation of stress intensity factor and $J$-integral, which shall provide better fracture analysis of the material. One can infer from the present results of plastic zones, $K_{I}$ and $J$-integral that the mid-plane of the specimen experiences smaller size of plastic zone size and higher magnitudes of $K_{I}$ or $J$ indicating higher out-of-plane constraint (centre of the specimen) than in-plane-constraint (at the surface). Due to higher out of plane constraint the material at the centre of the specimen thickness tends to fail earlier than that on the surface. 


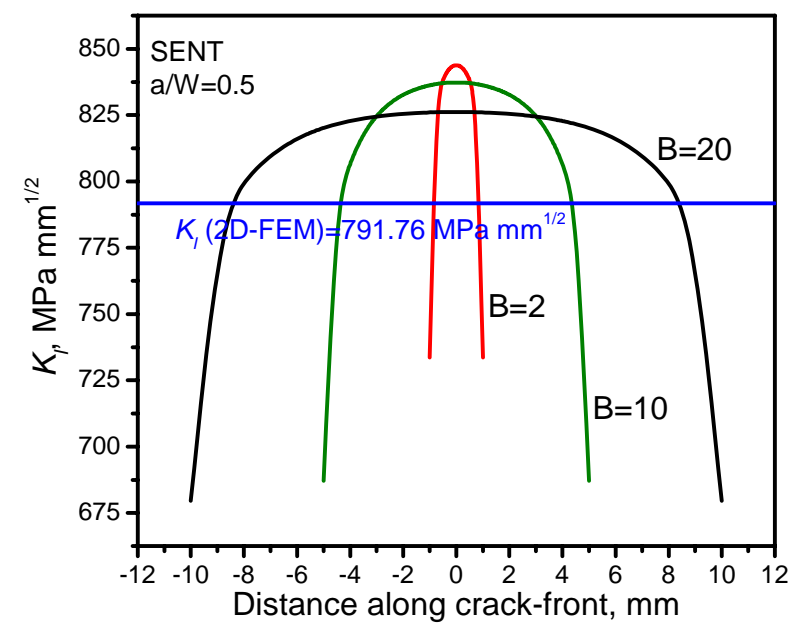

Figure 12. Variation of $K_{I} v s$. distance along the crack-front by elastic FEA

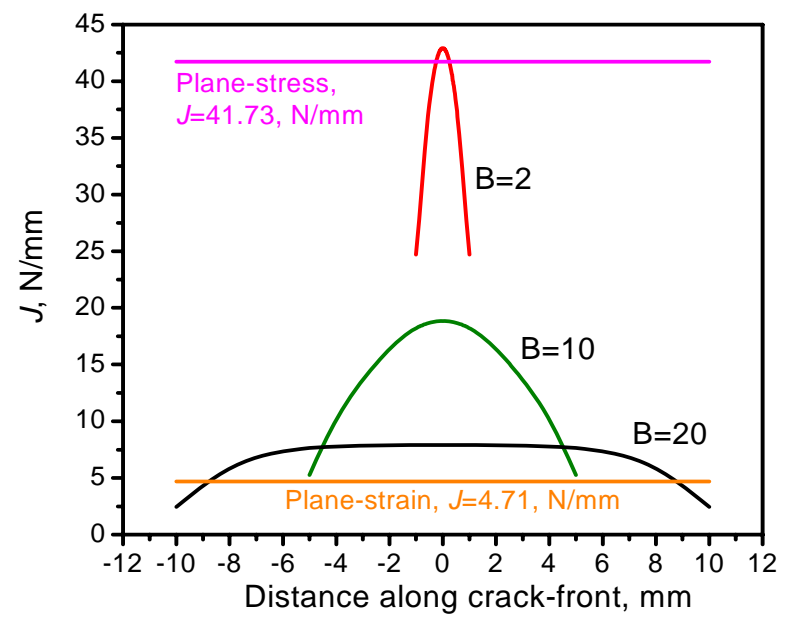

Figure 13. Variation of $J v s$. distance along the crack-front by elastic-plastic FEA

\section{Conclusions}

The following conclusions are made from the present study:

o By elastic-plastic analysis, unlike to elastic analysis, the estimated size of a plastic zone in the crack propagation direction is always higher on the surface than at the centre of a specimen in agreement with the natural expectation. The shapes of plastic zones obtained by both the elastic and elatic-plastic analyses do not match with classical dog-bone shape.

o The condition of specimen thickness $(B / W=0.5)$ laid by ASTM E399-83 to attain plane strain plastic zone size for the valid $K_{I C}$ fracture test is not observed from the results of elastic FEA for even $B=20 \mathrm{~mm}(B / W=1)$, this condition is closely met for elastic-plastic case.

o The ratio of the estimated plastic zone sizes obtained on surface and at the center of the specimen in case of elastic $\left(R_{e l}\right)$ and elastic-plastic $\left(R_{e l-p l}\right)$ analyses do not match with ratio of corresponding estimated 2D plane stress and plane strain $(R)$ results showing that the state of stress do not corresponding to plane stress on the surface and vice-versa for higher specimen thickness.

o The magnitude $K_{I}$ and $J$ varies along the crack-front; the variation depends on the specimen thickness. The magnitude of $K_{I}$ and $J$ are higher at the centre than the surface of the specimen, indicating higher out-of-plane constraint.

o Due to higher out-of-plane constraint conditions prevailing at the central zone of the crack front, the material instability is expected to start there rather than at the surface.

This 3D PZ study has clearly demonstrated that elastic-plastic FE analysis yields closer results to the ASTM requirements of materials $K_{I C}$ fracture testing rather than the elastic analysis as naturally expected. In future, this work can be extended to: (a) study 
the details of PZ for various loading conditions and various fracture test specimens geometries for more depth of clarity in specimen size requirements for a fracture toughness test, and (b) demarcating in-plane and out of-plane constraint effects in a fracture specimens.

\section{Nomenclature}

a crack length

$B \quad$ thickness of the specimen

$K_{I} \quad$ mode-I stress intensity factor

$K_{I C} \quad$ critical stress intensity factor

$J \quad$ J-integral

$r_{p} \quad$ plastic zone size along the crack-front $\left(\theta=0^{\circ}\right)$

$R \quad$ ratio of plane stress plastic zone size and plane strain plastic zone size estimated by 2D FE analysis

$R_{e l} \quad$ ratio of plastic zone size on the surface and at the centre of the specimen estimated by elastic 3D FE analysis

$R_{e l-p l} \quad$ ratio of plastic zone size on the surface and at the centre of the specimen estimated by elastic-plastic 3D FE analysis

$W \quad$ width of the specimen

$\sigma \quad$ applied stress

$\sigma_{y} \quad$ yield stress of the material

$v \quad$ Poisson's ratio

\section{abbreviations}

FE finite element

FEA finite element analysis

PZ plastic zone

PZS plastic zone size

SENT single edge notch tensile

2D two dimensional

3D three dimensional

\section{Acknowledgment}

Authors gratefully acknowledge the computational facilities provided by the Research Centre, B V B College of Engineering \& Technology, Hubli-580 031, India.

\section{References}

ABAQUS User’s Manual. Version 6.5-1. Hibbitt, Karlsson \& Sorensen, Inc: 2004.

ASTM E399-83, (1983). Standard Test Method for Plane-Strain Fracture Toughness (KIC) of Metallic Materials, Philadelphia.

ASTM E813-87, (1987). Standard Test Method for JIC, a Measure of Fracture Toughness, Philadelphia.

Buchholz F.-G., Chergui A., Richard H.A., 2004. Fracture analyses and experimental results of crack growth under general mixed mode loading conditions. Engineering Fracture Mechanics, Vol. 71, pp. 455-468

Coutin S., Gardin C., Bezine G., Ben Hadj Hamouda H, 2005. Advantages of the J-integral approach for calculating stress intensity factors when using the commercial finite element software ABAQUS. Engineering Fracture Mechanics, Vol. 72, pp. 2174-2185

Fernandez-Zuniga D., Kalthoff J.F., Fernandez-Canteli A., Grasa J., Doblare M., 2005. Three dimensional finite element calculations of crack-tip plastic zones and $\mathrm{K}_{\mathrm{IC}}$ specimen size requirements. ECF-15.

Gdoutos E., Papakalitakis G., 1987. Crack growth initiation in elastic-plastic materials. International Journal of Fracture, Vol.32, pp. 143-156.

Giner E., Fernandez-Zuniga D., Fernandez-Saez J., Fernandez-Canteli A., 2010. On the $J_{\mathrm{x} 1}$-integral and the out-of plane constraint in a 3D elastic cracked plate loaded in tension. International Journal of Solids and Structures, Vol.47, pp.934-946.

Gonzalez-Herrera, A., Garcia-Manrique, J., Cordero, A., Zapatero, J., 2006. Plastic zone study by means of tri-dimensional finite element models on fatigue crack closure, Key Engineering Materials, 324-325, Part 1, pp. 555-558

Irwin G., R., 1960. Plastic zone near a crack and fracture toughness. Mech. Metall. Behavior of Sheet Mater. Proc 7th Sagamore Ordinance Mater. Res. Conf., New York, Vol. 4, pp. 63-77.

Kodancha K., G., Kudari S., K., 2009. Variation of stress intensity factor and elastic T-stress along the crack-front in finite thickness plates. Frattura ed Integrità Strutturale, Vol. 8, pp. 45-51.

Kudari S., K., Maiti B., Ray K., K., 2007. The effect of specimen geometry on plastic zone size: a study using the J integral. Journal of Strain Analysis, Vol. 42, pp.125-136. 
Qu Jie, Wang Xin, 2006. Solutions of T-stresses for quarter-elliptical corner cracks in finite thickness plates subject to tension and bending. International Journal of Pressure Vessels and Piping, Vol. 83, pp.593-606.

Rajaram H., Socrate S., Parks D.M., 2000. Application of domain integral methods using tetrahedral elements to the determination of stress intensity factor. Engineering Fracture Mechanics, Vol. 66, pp. 455-482

Roychoudhury S., Dodds R., H., 2003. A numerical investigation of 3-D small-scale yielding fatigue crack growth, Engineering Fracture Mechanics, Vol. 70, pp. 2363-2383.

Subramanya H., Y., Viswanath S., Narasimhan R., 2007. A three-dimensional numerical study of mode I crack tip fields in pressure sensitive plastic solids. International Journal of Solids and Structures, Vol. 44, pp. 1863-1879.

Yuan H., Brocks W., 1998.Quantification of constraint effects in elastic-plastic crack front fields. J Mech Phys Solids, Vol. 46, pp. 219-241.

Zadeh G. M., Hardtke K. D., Wossidlo P., Wobst K., 1995. The importance of the triaxility modified J concept for the assessment of pressurised component with surface flaws. Nuclear Engineering and Design, Vol.157, pp.111-121.

Zhang Y., H., Edwards L., 1992. The effect of grain boundaries on the development of plastic deformation ahead of small fatigue cracks. Scripta Metallurgica, Vol. 26, pp. 1901-1906.

\section{Biographical notes}

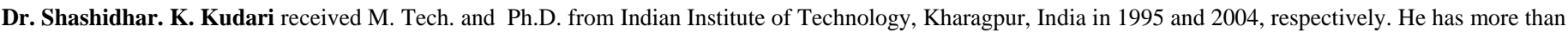

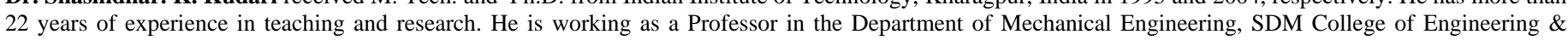

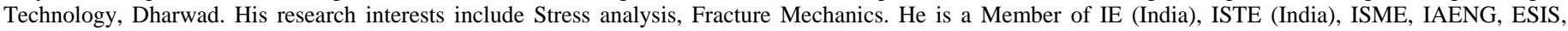
ISTAM. He has delivered several guest lectures in reputed Institutes in his research areas and has several technical publications to his credit.

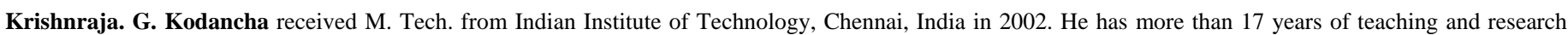

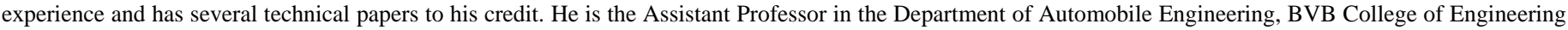
\& Technology, Hubli, India. . His research interests include stress analysis and fracture mechanics.

Received June 2010

Accepted August 2010

Final acceptance in revised form September 2010 